Sozial Extra 9|10 2012: 58-58 Dol 10.1007//12054-012-1018-2 Vorschau 11|12 '12

\section{Praxis aktuell: Junge Erwachsene in prekären Lebenslagen}

Den besonderen Lebenslagen junger Erwachsener wird im sozialwissenschaftlichen und fachlichen Diskurs Sozialer Arbeit verhältnismäßig wenig Aufmerksamkeit geschenkt -obwohl diese immer mehr von einer Prekarität von Übergängen und Ausgrenzungsrisiken geprägt sind, die Unterstützungsbedarfe mit sich bringen. Im Schwerpunkt lesen Sie Beiträge, die zum einen auf die besonderen Lebenslagen junger Erwachsener sowie zum anderen auf Soziale Arbeit mit dieser Zielgruppe eingehen: Wie reagiert der Sozialstaat auf prekäre Lebenslagen im jungen Erwachsenenalter, wer ist „zuständig“? Wie sehen heute Übergänge aus und welche innovativen Konzepte gibt es für Soziale Arbeit, diese zu begleiten und zu unterstützen? Wie erleben junge Erwachsene selbst ihre Lebenssituation und die Unterstützungsangebote sozialer Dienste?

\section{Durchblick: Jugend im Risikodiskurs:}

\section{Drogenkonsum zwischen Politik und Prävention}

Jugend und Drogenkonsum sind eng assoziiert. Gleichsam traditionell wird die Kriminalisierung des Umgangs mit Drogen durch eine besondere Gefährdung Jugendlicher legitimiert. Angesichts von Gesundheitsgefahren und Abhängigkeit stellt Prävention auf die Verhinderung von Drogenkonsum ab. Dass diese Sichtweise einseitig ist, wird seit längerer Zeit beklagt, und zuletzt kam wieder Bewegung in die Debatte. Allmählich wächst das Bewusstsein, dass Drogenkonsum bei Jugendlichen Teil ihrer „normalen“ Identitätsgestaltung sein kann und Verbote zusätzliche Anreize schaffen. In der Folge wird Prävention zunehmend als Unterstützung kontrollierten, risikobewussten Konsums konzipiert. Die Beiträge des Schwerpunktes setzen an dieser Stelle an und blicken von ihr auf Folgen für die Soziale Arbeit.

\section{Impressum \\ Sozial Extra}

Zeitschrift für Soziale Arbeit

Ausgabe 9|10 2012, 36. Jahrgang

ISSN 0931-279 X

VS Verlag | Springer Fachmedien Wiesbaden GmbH |

Abraham-Lincoln-Straße 46 | 65189 Wiesbaden | www.springer-vs.de

Amtsgericht Wiesbaden | HRB 9754 | USt-IdNr. DE811148419

Geschäftsführer: Dr. Ralf Birkelbach (Vors.) | Armin Gross | Albrecht F. Schirmacher

Director Sozialwissenschaften \& Forschungspublikationen: Dr. Reinald Klockenbusch

Director Ad Sales: Armin Gross

Director Marketing and Direct Sales: Rolf-Günther Hobbeling

Director Production: Christian Staral

REDAKTION

Verantwortlicher Redakteur: Detlef Ullenboom (i.S.d.P)

Sozial Extra, Hattinger Str. 551, 44795 Bochum, tel 0234 / 4388 947,

redaktion@sozialextra.de

Koordination „Praxis aktuell“: Rudolf Leiprecht

Koordination „Durchblick“: Roland Bader, Leonie Wagner

Redaktionsbeirat:

Kirsten Aner (Berlin), Gabriele Bingel (Berlin), Christoph Butterwegge (Köln), Margret Dörr (Mainz), Sabine Hering (Siegen), Peter Herrmann (Aghabullogue, Irland), Nadia Kutscher (Aachen), Richard Krisch (Wien), Regina Rätz (Berlin), Albert Scherr

(Freiburg), Wolfgang Schröer (Hildesheim), Helmuth Schweitzer (Duisburg), Werner

Thole (Kassel), Leonie Wagner (Holzminden), Margherita Zander (Münster).

VERLAG

Produktmanagement: Ronald Schmidt-Serrière

tel +49 (0)611 / $7878-280$, fax +49 (0)611 / 787878 - 280

ronald.schmidt-serriere@vs-verlag.de

Anzeigenleitung: Yvonne Guderjahn

tel +49 (0)611 / $7878-155$, fax +49 (0)611 / 7878 - 430

yvonne.guderjahn@best-ad-media.de

Anzeigendisposition: Monika Dannenberger

tel +49 (0)611 / 7878 - 148, fax +49 (0)611 / $7878-443$

monika.dannenberger@best-ad-media.de

Kundenservice:

Springer Customer Service Center GmbH I VS Verlag-Service

Haberstraße 7, D-69126 Heidelberg

Telefon: +49 (0)6221 / 345 - 4303 | Fax: +49 (0)6221 / 345 - 4229

Montag bis Freitag 8:00 Uhr bis 18:00 Uhr

vsverlag-service@springer.com

Anzeigenpreise: Es gelten die Mediadaten vom 01.01.2010.

(C) VS Verlag ist eine Marke von Springer DE. Springer DE ist Teil der

Fachverlagsgruppe Springer Science+Business Media.

Bezugsmöglichkeiten: Jährlich erscheinen 6 Doppelhefte. Jahresabonnement / privat (print+online) 66,- EUR; Jahresabonnement / privat (nur online) 55,- EUR; Jahresabonnement / Bibliotheken (nur Print) 168,- EUR; Jahresabonnement Institutionen/ Unternehmen (nur print) 118,- EUR; Jahresabonnement Studenten / Emeritus (print +online) - bei Vorlage einer Studienbescheinigung 39,- EUR. Alle Print-Preise zuzüglich Versandkosten. Jedes Abonnement Print und Online beinhaltet eine Freischaltung für das SozialExtraArchiv. Der Zugang gilt ausschließlich für den einzelnen Empfänger des Abonnements. Das Abo verlängert sich automatisch um ein Jahr, wenn es nicht 6 Wochen vor Ablauf des Bezugszeitraums beim Verlag gekündigt wurde. Jährlich können Sonderhefte erscheinen, die nach Umfang berechnet und den Abonnenten des laufenden Jahrgangs mit einem Nachlass von $25 \%$ des jeweiligen Ladenpreises geliefert werden. Bei Nichtgefallen können die Sonderhefte innerhalb einer Frist von 3 Wochen zurückgegeben werden.

\section{URHEBERRECHTE}

Die in dieser Zeitschrift veröffentlichten Beiträge und Abbildungen sind urheberrechtlich geschützt. Übersetzung, Nachdruck, Vervielfältigungen auf fotomechanischem oder ähnlichem Wege oder im Magnettonverfahren, Vortrag, Funk- und Fernsehsendung sowie Speicherung in Datenverarbeitungsanlagen (auch auszugsweise) nur mit Genehmigung des Verlags.

Von einzelnen Beiträgen oder Teilen von ihnen dürfen nur einzelne Kopien für den persönlichen oder sonstigen Gebrauch hergestellt werden.

Titelfoto: ( ) istockphoto.com

www.sozialextra.de

Beilagenhinweis:

Dieser Ausgabe von Sozial Extra liegt eine Beilage des Trauma Support GbR und Institut für Traumabearbeitung und Weiterbildung, Frankfurt, bei. Wir bitten um freundliche Beachtung. 